\title{
A linkage map of the Atlantic salmon (Salmo salar) based on EST-derived SNP markers
}

\author{
Thomas Moen*1,2, Ben Hayes ${ }^{1,2}$, Matthew Baranski1,2, Paul R Berg1,3, \\ Sissel Kjøglum ${ }^{4}$, Ben F Koop ${ }^{5}$, Willie S Davidson ${ }^{6}$, Stig W Omholt ${ }^{1,3}$ and \\ Sigbjørn Lien ${ }^{1,3}$
}

Address: ${ }^{1}$ CIGENE - Centre of Integrative Genetics, Ås, Norway, ${ }^{2}$ AKVAFORSK - Institute of Aquaculture Research, Ås, Norway, ${ }^{3}$ Institute of Animal and Aquacultural Sciences, Norwegian University of Life Science, Ås, Norway, ${ }^{4}$ Aqua Gen AS, Trondheim, Norway, ${ }^{5}$ Centre for Biomedical Research, Department of Biology, University of Victoria, Victoria, British Columbia, Canada and ${ }^{6}$ Department of Molecular Biology and Biochemistry, Simon Fraser University, Burnaby, British Columbia, Canada

Email: Thomas Moen* - thomas.moen@akvaforsk.no; Ben Hayes - ben.hayes@akvaforsk.no; Matthew Baranski - matt.baranski@akvaforsk.no; Paul R Berg - paul.berg@umb.no; Sissel Kjøglum - sissel.kjoglum@aquagen.no; Ben F Koop - bkoop@uvic.ca; Willie S Davidson - wdavidso@sfu.ca; Stig W Omholt - stig.omholt@umb.no; Sigbjørn Lien - sigbjorn.lien@umb.no

* Corresponding author

Published: 15 May 2008

BMC Genomics 2008, 9:223 doi:10.1/86/147|-2164-9-223
Received: 19 October 2007

Accepted: 15 May 2008

This article is available from: http://www.biomedcentral.com//47I-2/64/9/223

(C) 2008 Moen et al; licensee BioMed Central Ltd.

This is an Open Access article distributed under the terms of the Creative Commons Attribution License (http://creativecommons.org/licenses/by/2.0), which permits unrestricted use, distribution, and reproduction in any medium, provided the original work is properly cited.

\begin{abstract}
Background: The Atlantic salmon is a species of commercial and ecological significance. Like other salmonids, the species displays residual tetrasomy and a large difference in recombination rate between sexes. Linkage maps with full genome coverage, containing both type I and type II markers, are needed for progress in genomics. Furthermore, it is important to estimate levels of linkage disequilibrium (LD) in the species. In this study, we developed several hundred single nucleotide polymorphism (SNP) markers for the Atlantic salmon, and constructed male and female linkage maps containing SNP and microsatellite markers. We also investigated further the distribution of male and female recombination events across the genome, and estimated levels of LD between pairs of markers.
\end{abstract}

Results: The male map had 29 linkage groups and was $390 \mathrm{cM}$ long. The female map had 30 linkage groups as was 1983 cM long. In total, the maps contained 138 microsatellite markers and 304 SNPs located within genes, most of which were successfully annotated. The ratio of male to female recombination events was either close to zero or very large, indicating that there is little overlap between regions in which male and female crossovers occur. The female map is likely to have close to full genome coverage, while the majority of male linkage groups probably lack markers in telomeric regions where male recombination events occur. Levels of $r^{2}$ increased with decreasing inter-marker distance in a bimodal fashion; increasing slowly from $\sim 60 \mathrm{cM}$, and more rapidly more from $\sim 12 \mathrm{cM}$. Long-ranging LD may be consequence of recent admixture in the population, the population being a 'synthetic' breeding population with contributions from several distinct rivers. Levels of $r^{2}$ dropped to half its maximum value (above baseline) within $15 \mathrm{cM}$, and were higher than 0.2 above baseline for unlinked markers ('useful LD') at inter-marker distances less than $5 \mathrm{cM}$.

Conclusion: The linkage map presented here is an important resource for genetic, comparative, and physical mapping of the Atlantic salmon. The female map is likely to have a map coverage that is not far from complete, whereas the male map length is likely to be significantly shorter than the true map, due to suboptimal marker coverage in the apparently small physical regions where male crossovers occur. 'Useful LD' was found at inter-marker distances less than $5 \mathrm{cM}$. 


\section{Background}

The Atlantic salmon (Salmo salar) is a species of worldwide significance as a prized species in recreational fishing and a major contributor to the world's aquaculture production. The genomes of the Atlantic salmon and other salmonids are purported to be derivates of an autotetraploidisation event that occurred in their common ancestor 25 to 100 million years ago (reviewed in [1]). The subsequent re-diploidisation process is not yet complete, and is illustrated in such phenomena as duplicated DNA markers (e.g. [1]); the formation of tetravalent complexes during male meioses [2]; the apparent linkage of nonlinked loci due to non-random dissociation of the tetravalent complexes (pseudolinkage) [3,4]; unusual, partly tetrasomic, segregation patterns $[1,5-7]$; as well as chromosome arm numbers twice that of most other fin-fish species (reviewed in [8]). Furthermore, the formation of tetravalent complexes in males is believed to be the cause of another phenomenon observed in salmonids, very large differences in recombination rates between males and females that vary according to chromosomal region [6,9-13].

Two low-density maps have been published for Atlantic salmon [10,14], in addition to the SALMAP map, a higher-density, female microsatellite map made available online [15]. This latter map is developed from segregation data from two females from the river Tay in Scotland $[12,16]$, and contains 700 microsatellite type I and type II markers, out of which approximately 200 have been linked to BACs in the physical map [17]. Further progress in Atlantic salmon genomics relies on these existing maps being expanded with additional markers; in particular with Single Nucleotide Polymorphism (SNP) markers, since these are the most frequent polymorphisms in addition to being the markers of choice for high-throughput genotyping. Of particular value are SNP markers located within transcribed regions, to create more links between the genetic and physical maps.

Here, we provide an update of an ongoing project aiming at detecting, testing, and mapping large numbers of ESTderived SNP markers in Atlantic salmon [18,19]. We also present a $\mathrm{SNP} /$ microsatellite map to be used as a framework map, onto which additional SNP markers can later be added. Furthermore, we present more detailed results on sex-specific differences in recombination rates than has been provided before for this species. Finally, we report on levels of linkage disequilibrium (LD) between markers in the aquaculture strain from whence the mapping parents were sampled. Levels of LD vary between species and between populations [20-26], and have major implications for the feasibility of performing e.g. association studies and for fine-mapping Quantitative Trait Loci (QTL).

\section{Results}

\section{Detection and testing of SNP markers}

Hayes et al. [18] previously described the in silico detection of a large number of putative SNPs for Atlantic salmon, and the subsequent experimental testing of 86 of these SNPs in a diverse validation panel. In the present study, another set of 1369 SNP markers were tested in a similar validation panel (Table 1). Of the 1369 SNPs, 668 were polymorphic, reliably scored, and non-duplicated (Table 2). Of the 668 polymorphic, non-duplicated SNPs, 307 were chosen to be genotyped in the mapping families. These 307 SNPs had an average minor allele frequency of 0.27 , and an average observed heterozygosity of 0.29 (results for individual SNPs in Additional File 1). Out of 307 SNPs, 244 (79\%) resided in genes that could be identified and annotated (Additional File 1).

\section{Linkage map}

The mapping families, 10 full-sib families from a commercial breeding company, were genotyped for 307 SNPs and for 146 microsatellite markers. Out of the 307 SNP markers, 304 were polymorphic in at least one mapping parent. The SNPs belonged to 263 contigs, 222 of these having one SNP, 36 having two SNPs, and five having three SNPs.

All but four of the informative SNPs were integrated into the map, as were all 138 informative microsatellite markers. The male and female maps consisted of 29 and 30 linkage groups, respectively (Figures 1 to 3, Table 3, Additional File 2). The male map was $390 \mathrm{cM}$ long with 434 markers in total, while the female map was $1983 \mathrm{cM}$ long with 425 markers in total. Lengths of linkage groups ranged from $0 \mathrm{cM}$ to $59.7 \mathrm{cM}$ on the male map, and from

Table I: Samples used for SNP validation

\begin{tabular}{lll}
\hline Country & River/population & No of samples \\
\hline Canada & Conne & 3 \\
Canada & Placentia Bay & 3 \\
Iceland & Laxá & 3 \\
Iceland & Leirvogsá & 3 \\
Ireland & Moy & 3 \\
Ireland & Suir & 3 \\
Norway & Aqua Gena,b & 4 \\
Norway & Byglandsfjorden & 4 \\
Norway & Numedalslågen & 3 \\
Norway & SalmoBreeda & 3 \\
Norway/Finland & Tana-Jiesjokka & 3 \\
Russia & Neva & 3 \\
Russia & Varzuga & 3 \\
Spain & Asou & 3 \\
Spain & Pas & 3 \\
\hline
\end{tabular}

aAquacultural population

bThe Aqua Gen and Byglandsfjorden populations came in the form of $8 \mathrm{FI}$ hybrids between the two. 
Table 2: Experimental validation of SNPs

\begin{tabular}{lll}
\hline SNP type & No. & Freq. \\
\hline Normal & 668 & $48.8 \%$ \\
MSV & 47 & $3.4 \%$ \\
PSV & 67 & $4.9 \%$ \\
All homozygous & 379 & $27.7 \%$ \\
Failed & 208 & $15.2 \%$ \\
Total & 1369 & $100.0 \%$ \\
\hline
\end{tabular}

SNPs in the "normal" category were polymorphic, reliably scored and non-duplicated. MSV (multiple sequence variant) [49] SNPs were likely duplicated with polymorphism at one or both loci, PSV (paralogous sequence variants) SNPs were heterozygous in all animals, and thus likely duplicated without polymorphism. Failed SNPs displayed poor clustering of genotypes and/or unreliably scored genotypes.

$19.8 \mathrm{cM}$ to $117.1 \mathrm{cM}$ on the female map (Table 3 ). For the most part the same linkage groups were identified for the male and the female, with the following exceptions i) two linkage groups on the male map (s9 and s17) that each corresponded to two linkage groups on the female map, and ii) one linkage group on the female map (d21) that corresponded to two linkage groups on the male map. For 57 of all possible marker pairs informative in both sexes, the orientation of markers was not the same in the two sexes (Additional File 3). However, in all 57 cases the distance between markers on the male map was relatively small $(<1.7 \mathrm{cM})$, so that these differences most likely reflect upon minor genotyping errors or missing genotypes.

We did not observe in our data set any certain instances of pseudolinkage, the apparent linkage of non-linked chromosomes sometimes observed in male salmonids [5]. One likely homeologous relationship that has not been reported before [12] was found; marker Ssa418/i was located on LG24, while Ssa418/ii was located on LG16.

\section{Difference in recombination patterns between sexes and between parents}

Male recombination rates were much lower than female recombination rates in large parts of the genome. In some regions, however, male recombination rates were significantly higher than those of females. Invariably, these regions were located at the end of linkage groups (Figures 1 to 3). Ratios of male to female recombination fractions for adjacent markers tended to be either close to zero or very large (Figure 4). Of the male linkage groups, 16 had all their markers grouped into one very tight cluster, 11 had one large cluster of closely linked markers located at the end of the linkage group, and one had a large central cluster bordered by markers on either side (Figures 1 to 3 , Table 3).

\section{Linkage disequilibrium}

Levels of LD, measured through the correlation coefficient between pairs of loci $\mathrm{r}^{2}$, were calculated for all microsatellite-SNP pairs with minor allele frequency $(\mathrm{SNP})>0.2$ and heterozygosity (microsatellite) $>0.5$. The average $\mathrm{r}^{2}$ for marker pairs with markers located on different linkage groups was 0.16 . The average $\mathrm{r}^{2}$ for physically, but not genetically linked marker pairs (markers located more than $50 \mathrm{cM}$ apart on the same linkage group) was 0.20 . Levels of $\mathrm{r}^{2}$ increased with decreasing inter-marker distance from $\sim 60 \mathrm{cM}$, increased more rapidly more from $\sim 12 \mathrm{cM}$ (Figure 5), while $\mathrm{r}^{2}$ dropped to half its maximum value (above baseline) within $15 \mathrm{cM}$.

\section{Discussion}

Linkage map

The male map contained 29 linkage groups, corresponding well with the most common karyotype in European Atlantic salmon, which has $2 \mathrm{n}=58$ [27]. Most likely, therefore, each male linkage group corresponds to a separate chromosome. The female map, on the other hand, contained 31 linkage groups, including two pairs of linkage groups that each corresponded to a single male homologue. Each of these two pairs is likely to correspond to a single linkage group with a large segment not covered by markers.

The lack of markers in at least two segments of the female map shows that the female map is shorter than the true female genetic map. At the same time, the low number of female-informative markers that could not be mapped, in conjunction with the relative good fit of the map length with the length of the SALMAP map (1810 cM; [15]), indicates that the female map coverage most likely is not far from complete. The male map, on the other hand, may still be considerably shorter than the true male genetic map, due to recombination events apparently being localised to a small physical region (see below).

Map distances should be expected to be slight overestimates since any genotype errors would tend to inflate genetic distances. Although practically all genotypes resulting in double crossovers were re-checked in detail and corrected if necessary, genotype errors cannot be ruled out, in particular not for markers located at the end of linkage groups (where they cannot be revealed by testing for double crossovers).

Contrary to expectation, we did not observe any instances of pseudolinkage in the data set. Pseudolinkage describes the apparent linkage in male mapping parents of markers that are not linked in female mapping parents, with an excess of non-parental genotypes in offspring, and is believed to be caused by the non-random breaking up of tetravalent complexes formed during male meiosis [5]. 
Table 3: Properties of linkage groups

\begin{tabular}{|c|c|c|c|c|c|c|c|c|c|}
\hline \multirow[t]{2}{*}{ LG } & \multicolumn{4}{|c|}{ O meioses with $n$ recombination events } & \multirow[t]{2}{*}{$\begin{array}{l}\text { O map length } \\
\text { (cM) }\end{array}$} & \multirow[t]{2}{*}{$\begin{array}{l}\mathrm{O}^{7} \text { map length } \\
\text { (cM) }\end{array}$} & \multirow[t]{2}{*}{$\begin{array}{l}\text { Markers on } O^{7} \\
\text { map }\end{array}$} & \multirow[t]{2}{*}{$\begin{array}{l}\text { Markers on } 0^{x} \\
\text { map }\end{array}$} & \multirow[t]{2}{*}{$\begin{array}{l}0^{7} \text { map marker } \\
\text { clusters }\end{array}$} \\
\hline & $\mathrm{n}=0$ & $n=1$ & $n=2$ & $n \geq 3$ & & & & & \\
\hline I & 1118 & 687 & 75 & 0 & 103.8 & 1.21 & 6 & 6 & 1 \\
\hline 2 & 1090 & 671 & 108 & II & 68.4 & 0 & 26 & 23 & 1 \\
\hline 3 & 1473 & 390 & 17 & 0 & 87.6 & 23 & 15 & 14 & 2 \\
\hline 4 & 861 & 787 & 231 & I & 117.1 & 2.4 & 16 & 17 & I \\
\hline 5 & 1065 & 759 & 56 & 0 & 74.3 & 1.4 & 15 & 17 & I \\
\hline 6 & 1269 & 560 & 48 & 3 & 83.5 & 11.3 & 20 & 20 & 2 \\
\hline 7 & 1248 & 630 & 2 & 0 & 56.8 & 56.4 & 12 & 12 & 2 \\
\hline 8 & 1416 & 445 & 18 & 1 & 49.7 & 8.4 & 20 & 21 & 2 \\
\hline $90^{7}$ & NA & NA & NA & NA & NA & 32.6 & NA & 16 & 2 \\
\hline $9 \mathrm{a}+9$ & 1236 & 577 & 66 & 1 & 46.1 & NA & 12 & NA & NA \\
\hline 9bㅇ & NA & NA & NA & NA & NA & NA & 1 & NA & NA \\
\hline 10 & 1014 & 717 & 146 & 3 & 107.1 & 20.1 & 29 & 30 & 2 \\
\hline II & 1139 & 601 & 139 & 1 & 101.9 & 0 & 14 & 14 & I \\
\hline 12 & 767 & 907 & 206 & 0 & 99.3 & 10.8 & 18 & 18 & 2 \\
\hline 13 & 1589 & 291 & 0 & 0 & 29.4 & 12.6 & 13 & 29 & 2 \\
\hline 14 & 1273 & 581 & 25 & 1 & 40.2 & 57.8 & 12 & 12 & 2 \\
\hline 15 & 1638 & 242 & 0 & 0 & 38.4 & 1.5 & 9 & 9 & 1 \\
\hline 16 & $|66|$ & 214 & 5 & 0 & 43.2 & 4.6 & 9 & 9 & 2 \\
\hline $170^{7}$ & NA & NA & NA & NA & NA & 29.1 & NA & 15 & 3 \\
\hline 179 & $|87|$ & 9 & 0 & 1 & 2.5 & NA & 2 & NA & NA \\
\hline $17 q$ & 1458 & 300 & 111 & II & 109.4 & NA & 13 & NA & NA \\
\hline $18^{+}$ & 1417 & 431 & 32 & 0 & 65.8 & 58.3 & 9 & 9 & 2 \\
\hline 19 & 1691 & 189 & 0 & 0 & 26.8 & 1.2 & 6 & 6 & 1 \\
\hline 20 & 1586 & 282 & 12 & 0 & 56.5 & 0.5 & 9 & 9 & 1 \\
\hline 219 & 1625 & 240 & 15 & 0 & 41.4 & NA & 10 & NA & NA \\
\hline $21 \mathrm{a} \sigma^{7}$ & NA & NA & NA & NA & NA & 0 & NA & 1 & 1 \\
\hline $2 \mathrm{Ib} \sigma^{7}$ & NA & NA & NA & NA & NA & 0.1 & NA & 9 & I \\
\hline 22 & 969 & 862 & 47 & 2 & 68.3 & 0 & 15 & 14 & 1 \\
\hline 23 & 1242 & 595 & 40 & 3 & 98.7 & 1 & 16 & 15 & I \\
\hline 24 & 1247 & 561 & 69 & 3 & 103.7 & 0 & 9 & 9 & I \\
\hline 25 & 1263 & 595 & 22 & 0 & 55.2 & 1.3 & 12 & 12 & 1 \\
\hline 28 & 1334 & 513 & 32 & 1 & 102 & 18.2 & 13 & 14 & 2 \\
\hline 31 & 1815 & 65 & 0 & 0 & 29.2 & 3.2 & 5 & 5 & 1 \\
\hline 33 & 1494 & 374 & 9 & 3 & 56.2 & 32.5 & 9 & 9 & 2 \\
\hline$A$ & 1832 & 35 & 13 & 0 & 19.8 & 0.3 & 6 & 6 & 1 \\
\hline
\end{tabular}

OFemale-specific linkage groups

O'Male-specific linkage groups

Pseudolinkage has earlier been found in several salmonid mapping studies $[3,4,6,9,13]$. In a recent study on an F1 cross between Atlantic salmon of Canadian and European origin, utilising a subset of the SNP markers described in the present study, pseudolinkage was observed between 5 pairs of linkage groups (Boulding et al., submitted). The difference in occurrence of pseudolinkage between the latter study and the present study is in line with a hypothesis stating that pseudolinkage occurs more frequently in inter-strain hybrids than in pure strain fish $[5,9,28]$. It should be noted, however, that our findings with respect to pseudolinkage are indicative rather than conclusive since grandparental genotypes were not available, meaning that pseudolinkage could, if present, only be detected as weak linkage in males between markers unlinked in females (and not from excess of non-parental genotypes).
Also, since linkage phases were deduced from linkage analysis and not from grandparental genotyping, linkage between markers within male linkage groups could theoretically be due to pseudolinkage rather than to classical linkage. However, all markers on the male map were either i) very closely linked to other markers within the same linkage group, and hence very unlikely to be linked through pseudolinkage, or ii) appearing on the homologous linkage group in the female map. From this we conclude that pseudolinkage would be unlikely to be the cause of any observed linkages on this map.

\section{Difference in recombination patterns between sexes}

In salmonids, male recombination rates are much reduced compared to female recombination rates [9$11,14]$. In rainbow trout the ratio between male and 

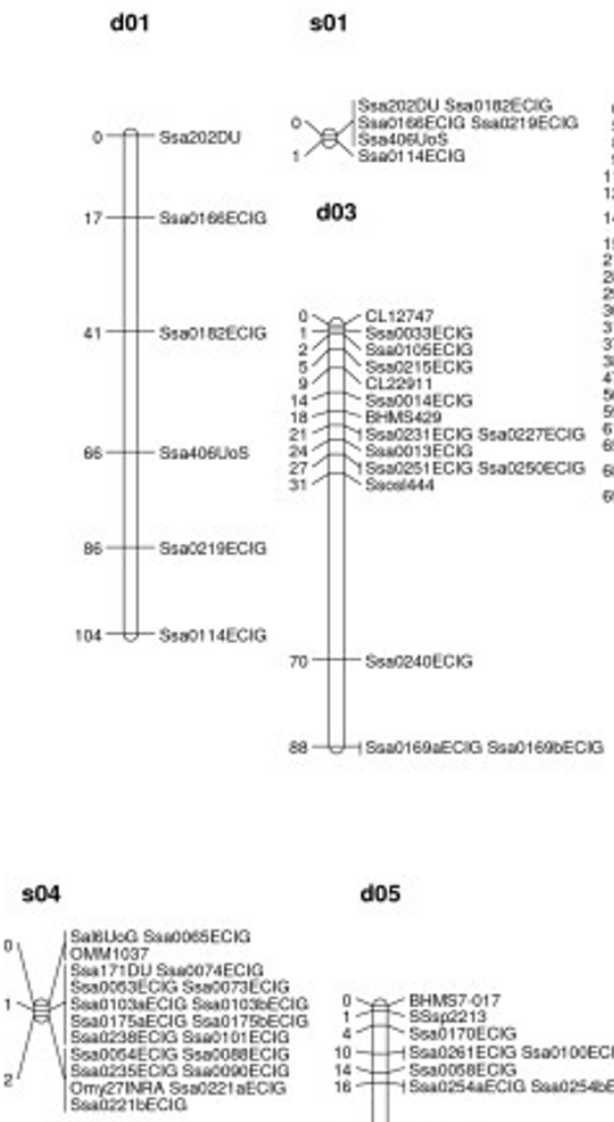

d06

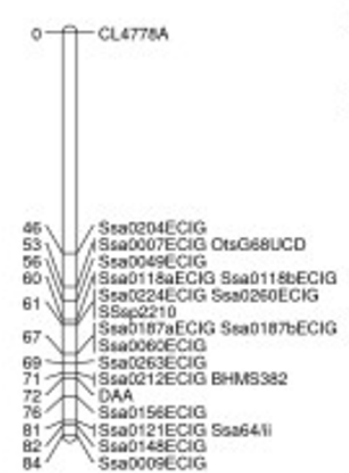

d05

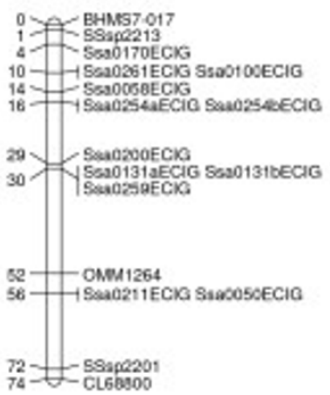

s07

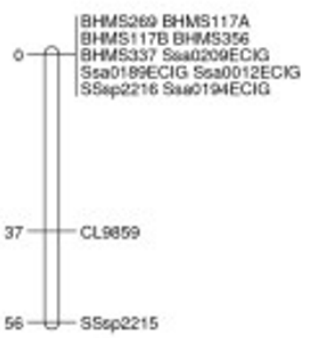

d02
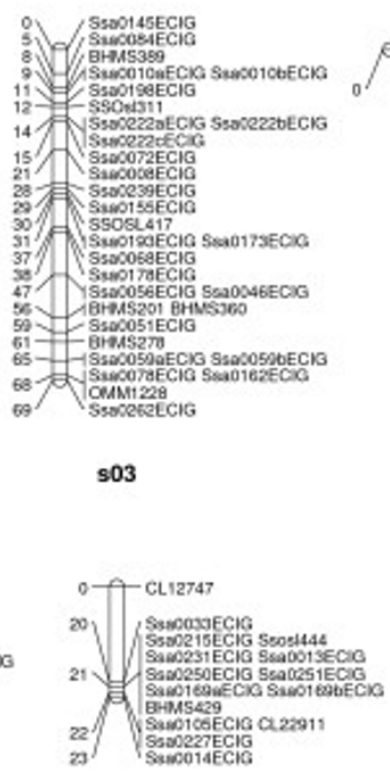

s05

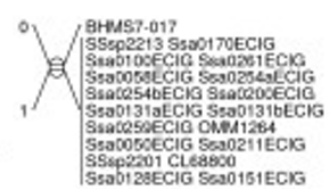

s06

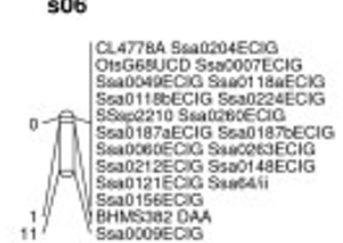

d08

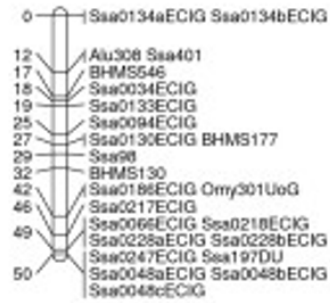

$s 02$

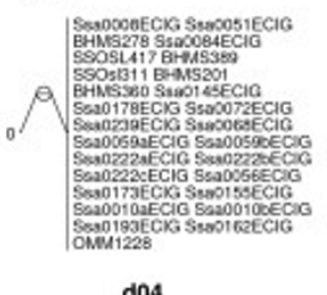

d04
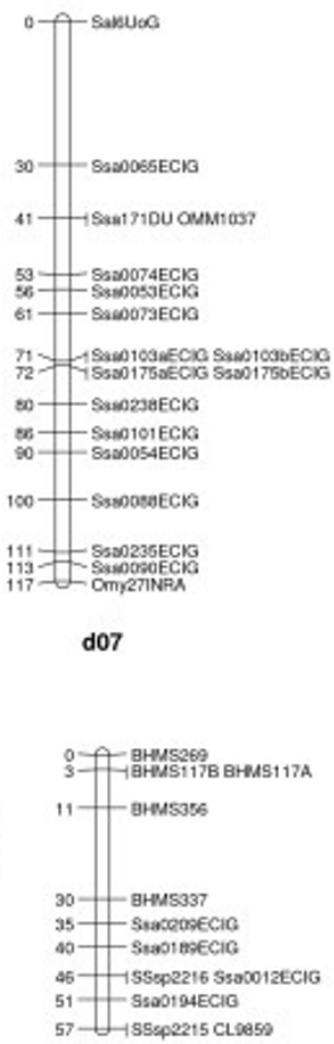

s08

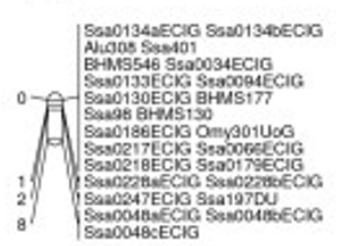

Figure I

male and female linkage maps for Atlantic salmon (linkage groups d0I to s08). Male and female linkage groups have prefixed s- and d-respectively. The linkage group nomenclature (numbers) is the same as in the map developed by the SALMAP project [15], except for one linkage group (sA/dA) that contains no markers present on the SALMAP map. The map units are Kosambi cM. 


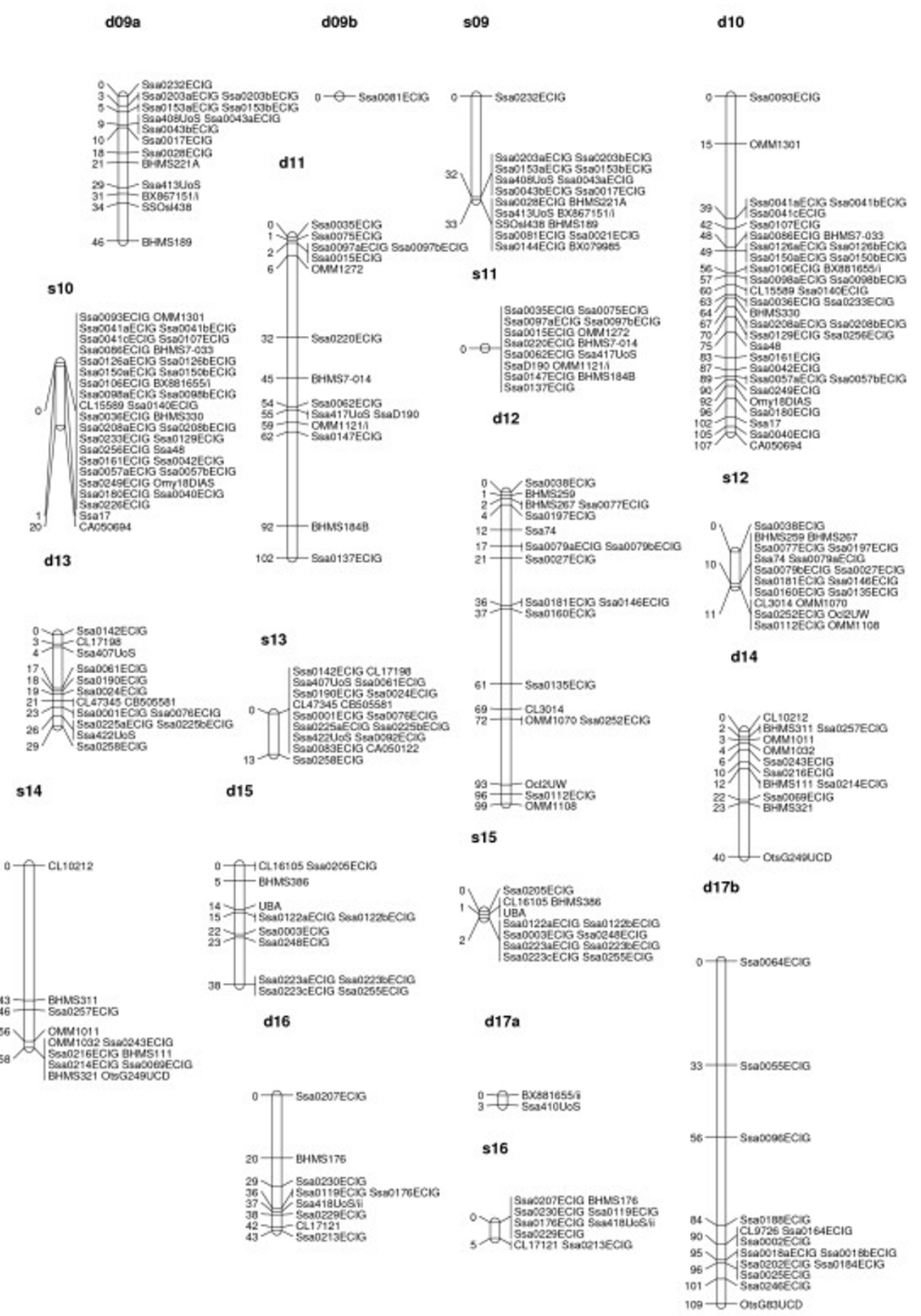

Figure 2

male and female linkage maps for Atlantic salmon (linkage groups d09a to s I 7b). Male and female linkage groups have prefixed s- and d-respectively. The linkage group nomenclature (numbers) is the same as in the map developed by the SALMAP project [I5], except for one linkage group $(\mathrm{sA} / \mathrm{dA})$ that contains no markers present on the SALMAP map. The map units are Kosambi cM. 
$s 17$

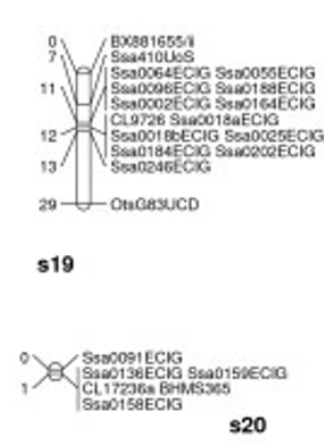

d20
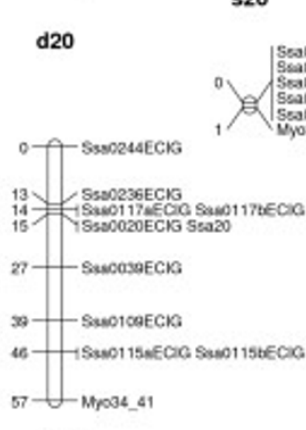

d22

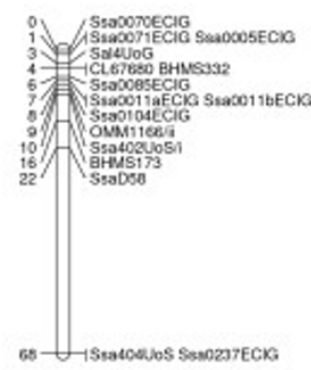

d28

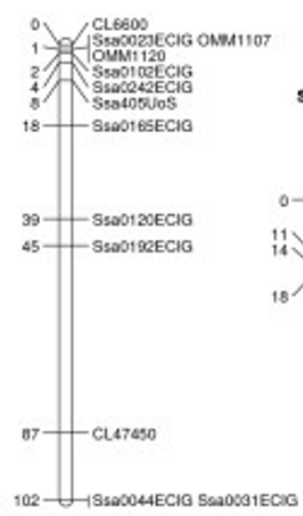

s28 d18

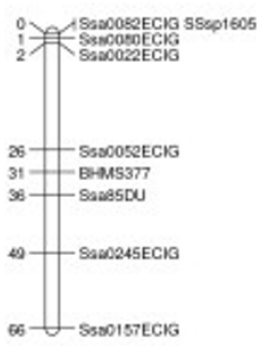

$\mathbf{s 1 8}$

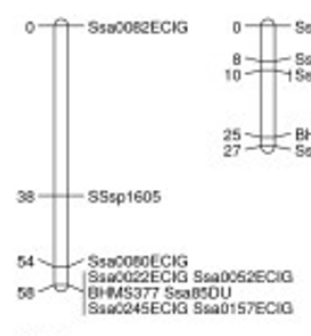

d19

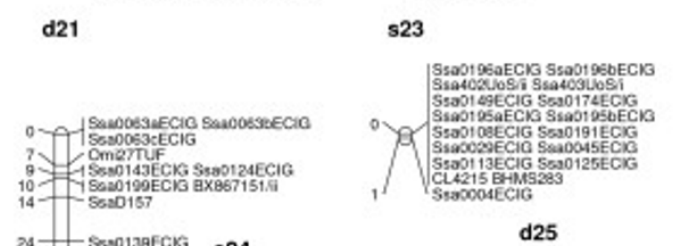

d25 s21a $\begin{aligned} & \text { OmarTUF Sano143ECIG } \\ & \text { S650124ECIG S500199ECIG }\end{aligned}$ SO20139ECIG SsaC019ECIO

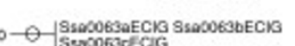

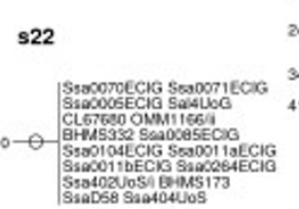

$24-$ SESO139ECIG $\$ 24$

d24

d23
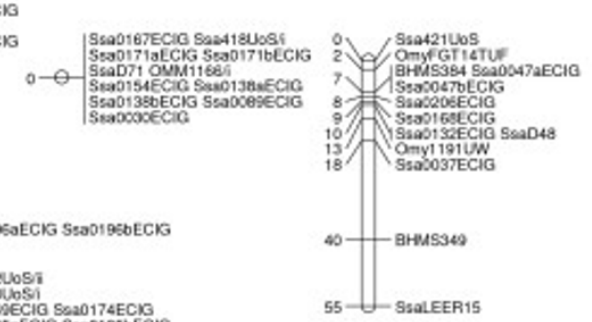

s25
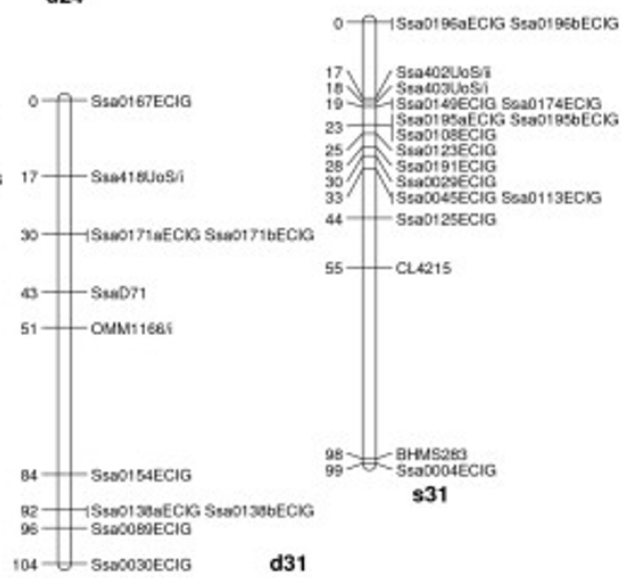
SEDO113ECIG $44-$ Ssa0125ECIG

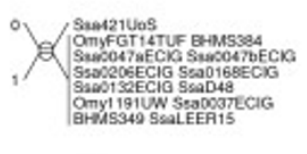

d33

d31

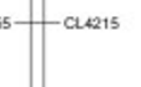

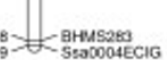

s31

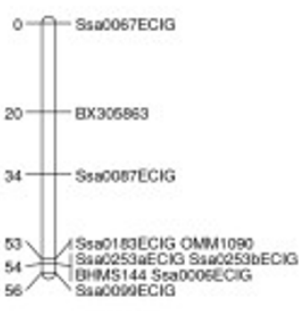

dA

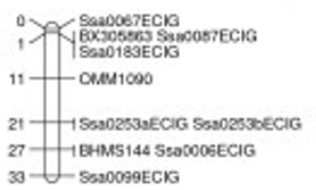

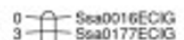

$8=$ TE200177ECIO 13 - TS620111AECIG Søa1116ECIG

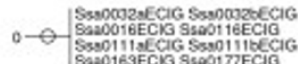

$20-$ Sen0116ECX

\section{Figure 3}

male and female linkage maps for Atlantic salmon (linkage groups s 17 to dA). Male and female linkage groups have prefixed s- and d-respectively. The linkage group nomenclature (numbers) is the same as in the map developed by the SALMAP project [15], except for one linkage group $(\mathrm{sA} / \mathrm{dA})$ that contains no markers present on the SALMAP map. The map units are Kosambi cM. 


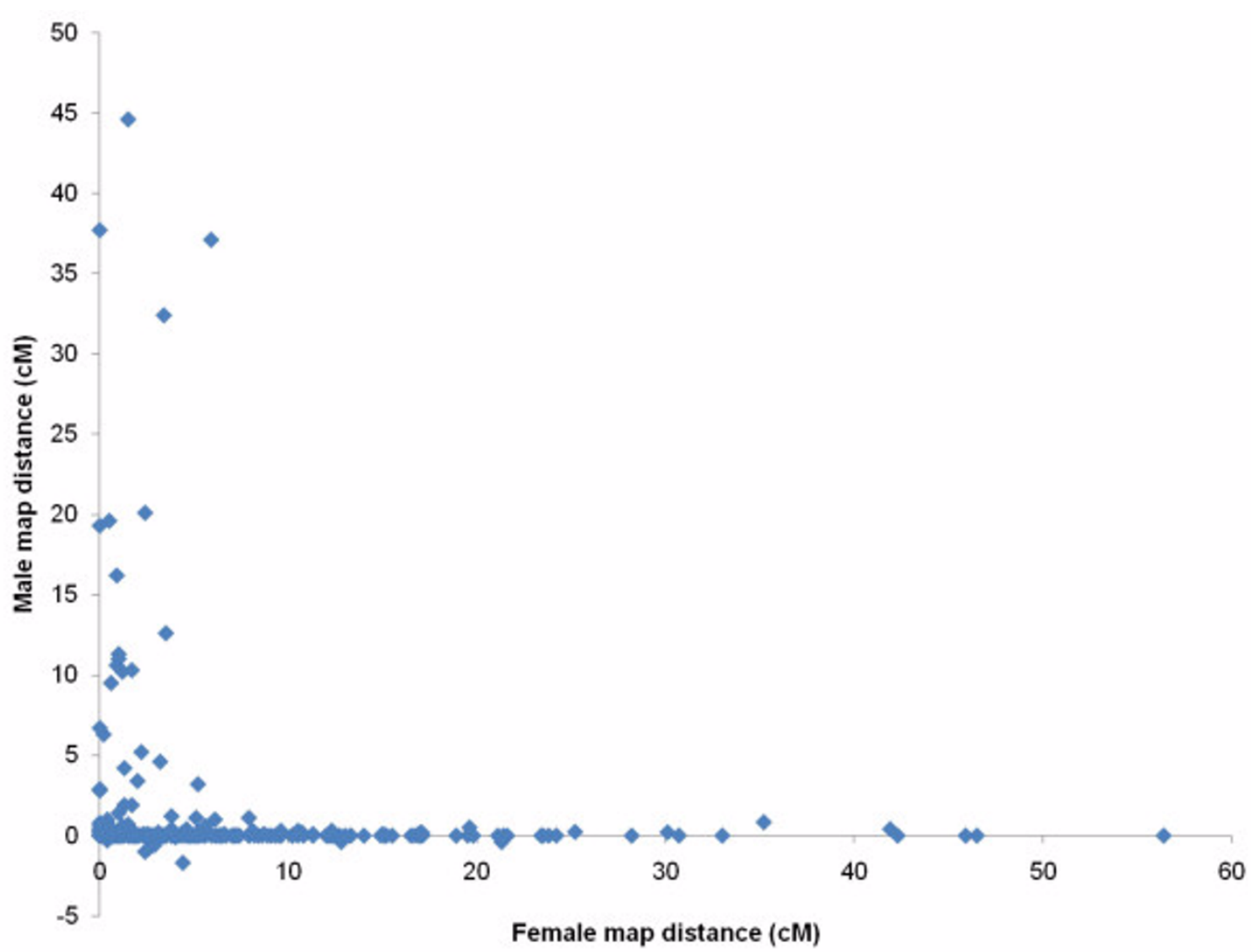

\section{Figure 4}

Male and female recombination rates for pairs of adjacent markers (d09a to s I 7b). Only markers on linkage groups with a I-to-I relationship between the male and female homologue were considered (i.e. linkage groups I7 and 2 I were excluded).

female recombination has been found to vary considerably across linkage groups, with male recombination rates being severely depressed relative to female recombination rates in areas proximal to centromeres, but elevated in regions distal to centromeres [9]. This finding has been investigated further in rainbow trout [12], and the same phenomenon has been shown in Arctic char [13] and Atlantic salmon $[10,29]$, though not in detail for the latter species. The results presented here support these earlier findings, but indicate that the site-specific differences in recombination rate between sexes are even more pronounced in Atlantic salmon than in rainbow trout (compare Figure 4 with Figure 2 from [9]). Since female recombination rates are much higher than male recombination rates for a large majority of adjacent marker pairs (Figures 1 to 3, Figure 4), it seems reasonable to assume that the regions where male recombination events occur are substantially smaller in physical terms than the corresponding female regions. This, again, means that, while it is likely that the female map presented here is close to full coverage, the true male map may be substantially longer than the presented male map. Higher marker densities will be needed to identify markers located in the regions where male recombination occurs, i.e. telomeric regions.

The most common karyotype of European Atlantic salmon consists of 16 metacentric and 42 acrocentric chromosomes [27]. If one assumes that male recombination occurs on all chromosomes, but only in telomeric regions, the complete male map should contain 8 linkage groups where a large central cluster of markers is surrounded by markers on both sides, and 21 groups with a large cluster at one end. In the map presented here, only linkage group 17 has a central cluster surrounded by markers on either side, indicating that the corresponding chromosome is one of the metacentric chromosomes. All other linkage groups have either only one cluster or one cluster with markers on one side, and thus may have one or two telomeric regions not covered by informative markers. Further evidence for the identification of linkage 


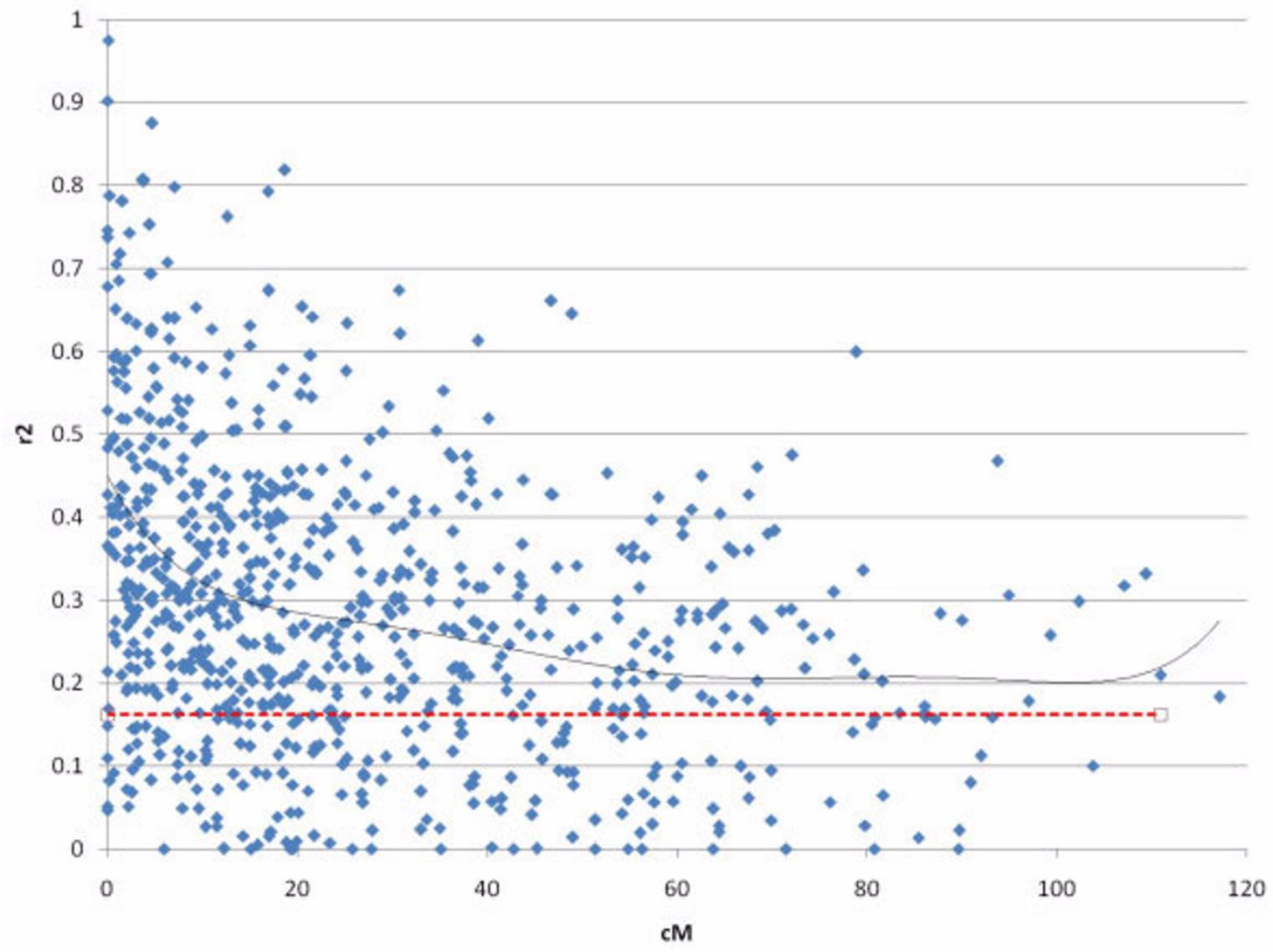

\section{Figure 5}

Levels of LD between microsatellite-SNP pairs located on the same linkage group plotted versus genetic distance. SNPs with minor allele frequencies $<0.20$ and microsatellites with heterozygosity $<0.50$ were excluded. Full line $=6^{\text {th }}$ degree polynomial best fit to the data; broken line $=$ average level of LD between physically unlinked markers (for comparison).

groups (i.e. chromosomes) as meta- or acrocentric could be extracted from the lengths of linkage groups (female map), and from the number of meioses with more than one recombination event (Table 3); if one assumes complete interference [30-33] then acrocentric chromosomes should have genetic lengths of $\sim 50 \mathrm{cM}$ and a small incidence of meioses with more than one crossover, while metacentric chromosomes should have genetic lengths of $\sim 100 \mathrm{cM}$ and a higher incidence of meiosis with two or more crossovers [12]. The linkage group size and number of meioses with $>2$ recombinants for linkage group 17 supports this hypothesis.

The site-specific distribution of male and female recombination events must be taken into account when QTL experiments are designed. In Atlantic salmon, it has become quite common to first perform a coarse genome scan using male segregation only and one or a few markers per linkage group, on the assumption that there is practically no recombination in males ([34]; Boulding et al., submitted). While this strategy will work for most parts of the genome, QTLs located in telomeric regions (which are also gene-rich regions) will probably be missed unless markers located in these regions are added. Identification of more markers in telomeric region would therefore be highly useful.

In this study, we exploited the lack of male recombination in large parts of the genome to draw more information out of the data. More specifically, for markers located in such regions, when both parents were heterozygous and identical within a given family, heterozygous (and thus, $a$ priori uninformative) offspring were assumed to have inherited the chromosome segment without recombinations from their respective fathers. In this way, alleles inherited from sire and dam could be deduced, and the markers could be re-coded as fully informative. It should be pointed out that this strategy was only used if no male 
recombinations had been observed in a priori informative offspring, meaning that no recombination had been observed in a minimum of 92 (but usually many more) meioses. The strategy was therefore conservative.

\section{Linkage disequilibrium}

The individuals used for the LD study were a subset of the mapping parents; more specifically, 16 mapping parents that belonged to the same breeding population, the remaining four animals belonging to another population. Phase-known data were used, since haplotypes could be deduced with very high certainty due to large family sizes.

Only microsatellite-SNP pairs were used for calculation of LD, thus mimicking the mapping of a QTL (usually assumed to be bi-allelic) using microsatellites. LD was also computed between SNP-SNP pairs (results not shown), and found to be much lower than for microsatellite-SNP LD. Levels of microsatellite-microsatellite LD were comparable to those of microsatellite-SNP LD, though slightly higher (results not shown). Lower LD values for SNP-SNP pairs when compared to microsatelliteSNP or microsatellite-microsatellite pairs is likely to be, at least in part, a consequence of differences in heterozygosity between marker types [35,36]. However, it could also be due in part to higher mutation rates of microsatellites relative to SNPs, which would impact upon levels of LD (reviewed in [37]). To avoid marker pairs with low information content, SNPs with minor allele frequencies below 0.2 and microsatellites with heterozygosities below 0.5 were culled from LD analysis.

The average $\mathrm{r}^{2}$ between markers located on different linkage group was higher than expected $\left(\mathrm{r}^{2}=0.16\right)$. This could be caused by limited effective population size and/or by relatedness between individuals in the sample (there were three pairs of full-sibs in the sample). However, removing closely related animals did not decrease, but rather increased $\mathrm{r}^{2}$ between unlinked markers, indicating that the small sample size may have been the main reason for high LD between unlinked markers. Levels of $\mathrm{r}^{2}$ is biased upwards when sample sizes are small, although much less so than the LD statistic D' [38]. At the same time, LD may have been slightly biased downward by the original detection of SNPs in EST sequences coming from a limited number of animals of both Canadian and Norwegian origin. Detection of SNPs in a small number of diverse animals could lead to an overrepresentation of old mutations among the set of SNPs, and thus to underestimation of LD between markers. It is difficult to assess the joint effect of these two factors. However, we have assumed that bias in levels of LD would not greatly affect conclusions regarding changes in $\mathrm{LD}$ with changing inter-marker distance.
LD between linked markers seemed to increase with decreasing inter-marker distance in a bi-modal manner. LD first increases slowly from $\sim 60 \mathrm{cM}$, then more rapidly from $\sim 12 \mathrm{cM}$. This finding may reflect upon the fact that the population from whence the haplotypes were derived is a 'synthetic' population, formed seven generation ago from individuals from different Norwegian rivers [39]. Due to the limited number of meioses since the formation of the base population (in this context less than four because only female crossovers would occur for most chromosomal regions), LD within linkage groups caused by population stratification would be expected to persist for long distances. Over shorter distances, LD would be caused both by population subdivision and by LD inherent in the wild population from whence the breeding population was formed. Since LD observed in the population is likely to be partly caused by population admixture, the results described here may not be relevant for wild Atlantic salmon populations. However, many breeding populations, and thus populations used for scanning of QTL/genes affecting commercial traits, are admixed in the same sense, and may thus display similar patterns of LD.

Levels of LD were calculated in order to estimate the marker densities needed to perform LD-based mapping in Atlantic salmon. The measure $r^{2}$ is equal to the amount of information provided by one locus about the other, meaning that for a gene-trait association to be detected, sample size must be increased by $1 / \mathrm{r}^{2}$ if a marker in $\mathrm{LD}$ with the trait-affecting gene is used rather than the gene itself. Levels of $\mathrm{r}^{2}$ from 0.1 to 0.3 have been proposed as minimum values of 'useful ld' $[40,41]$, in which case sample sizes would have to be increased by a factor of three to 10 in order to achieve maximum power. If one accepts $r^{2}$ $>0.2$ as the threshold, 'useful LD' could in this study be found at inter-marker distances below $\sim 5 \mathrm{cM}$, indicating that 400 fully informative, evenly spaced markers would be sufficient to at least start capturing inherent LD information. Thus, currently available maps ([15]; present study) could in principle provide a template for LD mapping to some extent. To fully exploit LD in the Atlantic salmon genome, however, more dense maps would probably be needed.

\section{Conclusion}

In this study, we constructed male- and female genetic linkage maps of Atlantic salmon, elucidated further the distributions of recombination events in males and females, and provided initial data on levels of linkage disequilibrium. The female map presented here is likely to represent the true genetic map well, whereas the male map is probably incomplete due to male recombination being localised to narrow telomeric regions. There appear to be little overlap between regions in which male and female recombination events occur. Levels of $\mathrm{LD}\left(\mathrm{r}^{2}\right)$ were more 
than 0.2 above the baseline for unlinked markers at intermarker distances less than $5 \mathrm{cM}$. At inter-marker distances larger than $15 \mathrm{cM}, \mathrm{r}^{2}$ decreased slowly, possibly reflecting LD due to population admixture that have had limited time to be broken down by recombination. The map presented here will serve as a framework map onto which a larger number of SNP markers, currently being identified from alignment of EST sequences and from DNA resequencing [42], will be added.

\section{Methods \\ SNP discovery}

Putative SNP were discovered in silico, as described in [18]. In brief, 100,866 EST chromatograms were base-called and aligned using the software programs Phred [43] and Phrap (P. Green, unpublished), whereupon putative SNPs were identified using PolyBayes [44] and checked by manual inspection. The ESTs were derived from several individuals of the Canadian McConnell strain and from several individuals from the Aqua Gen strains $[18,45,46]$.

\section{Validation and characterisation of SNPs}

From among the 2507 putative SNP discovered in silico, 1369 were chosen to be experimentally validated based on read quality and number of reads with rare allele. These SNPs were genotyped in a panel of 47 Atlantic salmon from across the species range (Table 1). SNP genotyping was done using the MassARRAY system from Sequenom (San Diego, USA). PCR-primers and extension-primers were designed using the software SpectroDESIGNER v3.0 (Sequenom). Multiplexes and primer sequences are available on request sigbjorn.lien@umb.no. Multiplexing levels were between 20 and 29. All SNP genotyping was performed according to the iPLEX protocol from Sequenom (available at [47]). For allele separations the Sequenom MassARRAY ${ }^{\mathrm{rm}}$ Analyzer (Autoflex mass spectrometer) was used. Genotypes were assigned in real time [48] by using the MassARRAY SpectroTYPER RT v3.4 software (Sequenom) based on the mass peaks present. All results were manually inspected, using the MassARRAY TyperAnalyzer v3.3 software (Sequenom). Classification of SNPs was based on this manual inspection. The categories were: 1) normal = polymorphic, reliably scored and non-duplicated; 2) $M S V$ multiple sequence variant) [49] = SNPs were likely duplicated with polymorphism at one or both loci (characterised by heterozygote excess, more than one cluster of heterozygotes, and presence of homozygotes); 3) PSV (paralogous sequence variant) = duplicated SNP without homozygotes; 4) all homozygous = all animals were homozygous; and 5) failed assay = poor clustering of genotypes and/or unreliably scored genotypes. Sequences of SNPs and contigs can be found in Additional File 1.

\section{Annotation of SNPs}

Sequences containing SNPs were clustered into contigs using a two stage Phrap assembly process. The first stage assembly of 434,384 Atlantic salmon EST sequences (parameters 100 minmatch and 0.99 repeat_stringency) resulted in 119,912 contigs which were then reassembled (2nd stage; 300 minmatch and 0.96 repeat_stringency) into 81,398 contigs [50]. Complete contigs containing SNPs compared (BLASTX) to CDD and Swissprot databases and annotated with the top BLASTX hit if the database match had an e-value of $<10^{-10}$. Matches to hypothetical proteins and genomic sequences were filtered out.

\section{Genotyping of mapping population}

The mapping families were provided by the breeding company Aqua Gen AS, and were used also for QTL mapping for resistance against the viral disease Infectious Pancreatic Necrosis (IPN) (Moen et al., in prep.). Hence, the offspring had been challenge tested for resistance to IPN. The 20 mapping parents came from two different year populations of Aqua Gen salmon; 16 from year class 2001, and 4 from year class 2000. Both populations were formed in the yearly 1970's from salmon from different Norwegian rivers [39], and have been maintained as more or less closed populations since (with increase in inbreeding per generation < 0.5\%; S. Kjøglum, Aqua Gen, pers. com.). In total, 192 offspring from each of 10 full-sib families, and their parents, were genotyped. The parents of the mapping population can, in the context of the present study, be regarded as random animals sampled from the broodstock population. DNA extraction was carried out using the DNAeasy 96 kit from QIAGEN (Venlo, the Nethelands). Within each family, microsatellites were genotyped for the 96 least and the 96 most resistant animals. SNPs were genotyped on the mapping families for two reasons; 1) to position the SNPs on the linkage map, and 2) to provide more markers for eventual fine-mapping of QTL-regions. In order to achieve these two goals in an affordable manner, we chose to genotype all SNPs for IPN-resistant animals only. The animals were genotyped for 307 SNP markers and for 148 microsatellites. SNP genotyping was carried out as described above.

Most of the microsatellite markers used in this study were chosen from the SALMAP microsatellite map of Atlantic salmon (as it was in 2006) [15], and were collectively chosen to ensure good genome coverage. The microsatellite markers were distributed across 32 PCR multiplexes that were subsequently combined into 16 multiplexes for capillary electrophoresis. Primer sequences and multiplex information is available on request. Polymerase chain reactions (PCR) were performed in volumes of $5 \mu \mathrm{l}$, using 0.25 units of AmpliTaq Gold (Applied Biosystems), 250 $\mu \mathrm{M}$ dNTP mix, $1.5 \mathrm{mM} \mathrm{MgCl}_{2}, 0.25-1$ pmol of each 
primer (depending on amplification efficiency of each marker in multiplex), $0.25 \mu \mathrm{l}$ DMSO, and $5 \mathrm{ng}$ DNA template. PCR cycling conditions were $95^{\circ} \mathrm{C}$ for 10 minutes, 35 cycles of $94^{\circ} \mathrm{C}$ for 30 seconds, $54^{\circ} \mathrm{C}$ for 1 minute, and $72^{\circ} \mathrm{C}$ for 1 minute, followed by a final extension of $60^{\circ} \mathrm{C}$ for 45 minutes. The lengths of the fluorescent PCR products were determined relative to the LIZ500 size standard (Applied Biosystems) on a 3730 DNA Analyzer (Applied Biosystems), using GeneMapper 4.0 (Applied Biosystems) software for allele calls.

\section{Construction of linkage map}

Since recombination rates in salmonids have been shown to differ dramatically between sexes, separate male and female maps were constructed. Marker grouping and initial marker ordering was done in Joinmap 3.0 [51]. A Joinmap 3.0 input file was made for each mapping parent (in double haploid format), containing information on alleles inherited from that parent only. Marker grouping was done at a minimum LOD score of 4.0. Following marker grouping, homologous linkage groups from each sire and each dam were integrated into single sex-specific maps. The marker orders determined by Joinmap 3.0 were tested and corrected using the flips function of CRIMAP, with a moving window of 7 markers (flips7). Using the final marker orders as calculated by CRIMAP, the data was examined for unlikely double recombinants, for inconsistencies in marker order between parents, using a custom Visual Basic for Applications (VBA) for Excel program. Segregation distortion was tested for using the same program, by incorporating a Pearson's goodness-offit test for 1:1 segregation of alleles from individual parents to offspring. Double recombinants occurring over small distances were checked for genotyping error. Markers displaying segregation distortion $(\mathrm{P}<0.01)$ were also inspected. After marker orders and potential genotype errors had been verified, the final maps were constructed using the fixed function of CRIMAP. The Kosambi mapping function was used. Map drawings were made using Joinmap 3.0 [51].

Since SNPs are bi-allelic, there were frequent occurrences of both parents of a family being heterozygous for the same two alleles of a SNP. In such cases, all heterozygous offspring were initially uninformative for mapping. We exploited the complete lack of male recombinants in most parts of the genome to deduce the inheritance of alleles in situations where i) both parents were heterozygous for the same two alleles of a SNP (marker A), and ii) the SNP was linked without any observed male recombinants to another marker (marker B); the latter marker being fully informative in the male. The steps of the deduction process were 1) determine the linkage phase between $\mathrm{A}$ and $\mathrm{B}$ in the male parent, 2) use the linkage phase to deduce which allele was inherited from the father at $\mathrm{A}$, to off- spring heterozygous at that marker, and 3) assign the other allele at $\mathrm{A}$ (of heterozygous offspring) to the female parent. This strategy was applied during the process of checking the data, automated through a VBA program.

Before map construction, SNP markers located within the same contig were combined to produce one marker point (i.e. a haplotype).

Linkage groups were numbered according to the SALMAP map, using shared microsatellites to infer homologies. Whenever two or more markers were shared between a linkage group and its SALMAP homologue, the linkage group was also oriented in the same way as the SALMAP linkage group. One linkage group did not share any markers with any linkage group on the SALMAP map, and was termed "A". In the cases where two unlinked (in this data set) female segments corresponded to a single male homologue $\mathrm{X}$, the segments were designated $\mathrm{Xa}$ and $\mathrm{Xb}$ to indicate that they were likely to be part of the same linkage group (and vice versa for unlinked male segments corresponding to a single female homologue).

The chrompic function of CRIMAP was used to count number of recombination events per meiosis.

\section{Linkage disequilibrium}

The animals used for calculation of LD were a subset of the mapping parents, more specifically the 16 parents that came from the breeding population of major representation. Thus, the data set consisted of 32 haplotypes. Only microsatellite-SNP pairs were used, to mimic the mapping of a QTL using microsatellite markers. SNPs with minor allele frequencies $<0.2$ and microsatellites with heterozygosities $<0.5$ were culled. Composite SNP markers (haplotypes of SNPs within the same contigs) were grouped with microsatellites. The haplotypes of the mapping parents were deduced at every linkage group using a custommade VBA program. Briefly, the program performed these steps (for every linkage group within every mapping parent): 1) Start at the first informative marker from one of the linkage group; 2) find the linkage phase between that marker and the next informative marker, minimising the number of recombination events in the offspring; 3 ) proceed in this manner to find the linkage phase between all informative marker, and thus to build the two haplotypes; and 4) for monomorphic markers, insert the same allele in both haplotypes. Measures of LD were calculated using the function haploxt of the program GOLD [52]. The LD measure was the correlation coefficient, $\mathrm{r}^{2}$, calculated as the square of Cramer's V [53]. The sampling effect was corrected for by subtracting 1 (number of haplotypes) from $r^{2}$ [54]. Sved's equation [55] was fitted to the data using the nlinfit function of MATLAB, but provided a poor fit (as expected, since LD in the population would be expected to 
be caused by other factors in addition to drift). Instead, a $6^{\text {th }}$ degree polynomial was fitted to the data.

\section{Authors' contributions}

TM took part in microsatellite genotyping and SNP validation, did data analyses except for SNP detection and annotation, and wrote a draft of the manuscript with contributions from $\mathrm{BH}, \mathrm{MB}, \mathrm{PB}, \mathrm{BK}, \mathrm{WSD}, \mathrm{BFK}$, and SL. $\mathrm{BH}$ did in silico SNP detection. MB coordinated microsatellite genotyping and took part in SNP genotyping. PB coordinated SNP genotyping. SK organised and carried out tissue sampling. BFK took part in annotation and provided the bulk of EST sequences. WSD took part in annotation. SWO provided laboratory facilities and took part in planning the study. SL provided laboratory facilities, did manual inspection of SNP validation data, and took part in SNP validation and data collection. All authors read and approved of the manuscript.

\section{Additional material}

\section{Additional file 1 \\ Properties of SNP markers used in the study. Excel workbook containing IDs of SNPs, contigs sequences $w / S N P$ sequence and position, allele fre- quencies, heterozygosities, and BLASTX hits \\ Click here for file \\ [http://www.biomedcentral.com/content/supplementary/1471- \\ 2164-9-223-S1.xls] \\ Additional file 2 \\ Atlantic salmon linkage map. Linkage map in Excel format. \\ Click here for file \\ [http://www.biomedcentral.com/content/supplementary/1471- \\ 2164-9-223-S2.xls] \\ Additional file 3 \\ Marker pairs with male-female order discrepancies. Marker pairs for which marker order were inverted in one map relative to the other. Click here for file \\ [http://www.biomedcentral.com/content/supplementary/1471- 2164-9-223-S3.xls]}

\section{Acknowledgements}

We wish to thank Hege Munck, Katrine Hånes, and Mariann Árnyasi for technical assistance; Bjørn Høyheim for proving EST sequences; Elin Bergseth, BioBank AS (Hamar, Norway), and VESO Vikan (Namsos, Norway) for preparation of samples; Aqua Gen Ltd., Dr. Kjetil Hindar, Dr. Tom Cross, Dr. lan Fleming, Dr. Guðni Guðbergsson, Dr. Sonia Consuegra and Dr. Arne Jenssen for providing tissue samples; and Dr. Roy Danzmann for providing access to the SALMAP map and microsatellite primer sequences. This study was funded by The Norwegian Research Council (NFR FUGE grant to CIGENE and NFR Havbruk \#169522/S40), by Genome Canada and Genome British Colombia (through cGRASP), and by Aqua Gen Ltd. None of the funding bodies had any role in the collection, analysis, or interpretation of data; in the writing of the manuscript; or in the decision to submit the manuscript for publication.

\section{References}

I. Allendorf FW, Thorgaard GH: Tetraploidy and the evolution of salmonid fishes. In Evolutionary Genetics of Fishes Edited by: Turner B]. New York, Plenum Press; 1984: I-46.

2. Svardson G: Chromosome studies of salmonidae. Rep Swed State Inst Fresh-Water Fish Res 1945, 23: I- I 5 I.

3. Wright JE, May B, Stoneking M, Lee GM: Pseudolinkage of the duplicate loci for supernatant aspartate aminotransferase in brook trout, Salvelinus fontinalis. J Hered 1980, 7 I:223-228.

4. May B: The salmonid genome: Evolutionary restructuring following a tetraploid event. PhD thesis. Volume Ph.D. Pennsylvania State University; 1980.

5. Wright JE, Johnson K, Hollister A, May B: Meiotic models to explain classical linkage, pseudolinkage, and chromosome pairing in tetraploid derivative salmonids genomes. Isozymes Curr Top Biol Med Res 1983, 10:239-260.

6. Johnson $\mathrm{K}$, Wright JE, May $\mathrm{B}$ : Linkage relationships reflecting ancestral tetraploidy in salmonid fish. Genetics 1987, | | 6:579-59|.

7. Allendorf FW, Danzmann R: Secondary tetrasomic segregation of MDH-B and preferential pairing of homeologues in rainbow trout. Genetics 1997, I45:1083-1092.

8. Phillips RB, Rab P: Chromosome evolution in the Salmonidae (Pisces): an update. Biol Rev (Camb) 200I, 76:I-25.

9. Sakamoto T, Danzmann R, Gharbi K, Howard P, Ozaki A, Khoo SK, Woram RA, Okamoto N, Ferguson MM, Holm LE, Guyomard R, Høyheim B: A microsatellite linkage map of rainbow trout (Oncorhynchus mykiss) characterized by large sex-specific differences in recombination rates. Genetics 2000, | 55: | 33 |- | 345 .

10. Moen T, Høyheim B, Munck H, Gomez-Raya L: A linkage map of Atlantic salmon (Salmo salar) reveals an uncommonly large difference in recombination rate between the sexes. Animal Genetics 2004, 35(2):81-92.

II. Woram RA, McGowan C, Stout JA, Gharbi K, Ferguson MM, Høyheim B, Davidson EA, Davidson WS, Rexroad C, Danzmann R: A genetic linkage map for Arctic char (Salvelinus alpinus): evidence for higher recombination rates and segregation distortion in hybrid versus pure strain mapping parents. Genome 2004, 47:304-315.

12. Danzmann R, Cairney M, Davidson WS, Ferguson MM, Gharbi K, Guyomard R, Holm LE, Leder E, Okamoto N, Ozaki A, Rexroad CE, Sakamoto T, Taggard JB, Woram RA: A comparative analysis of the rainbow trout genome with 2 other species of fish (Arctic charr and Atlantic salmon) within the tetraploid derivative Salmonidae family (subfamily: Salmoninae). Genome 2005, 48: |037-105I.

13. Gharbi K, Gautier A, Danzmann R, Gharbi K, Sakamoto T, Høyheim B, Taggard JB, Cairney M, Powell R, Krieg F, Okamoto N, Ferguson $\mathrm{MM}$, Holm LE, Guyomard R: A linkage map for brown trout (Salmo trutta): chromosome homeologies and comparative genome organization with other salmonid fish. Genetics 2006 , i 72:2405-2419.

14. Gilbey J, Verspoor E, McLay A, Houlihan D: A microsatellite linkage map for Atlantic salmon (Salmo salar). Animal Genetics 2004, 35:98-105.

15. ASalBase [http://www.asalbase.org]

16. Woram RA, Gharbi K, Sakamoto T, Hoyheim B, Holm LE, Naish K, McGowan C, Ferguson MM, Phillips RB, Stein J, Guyomard R, Cairney M, Taggart JB, Powell R, Davidson W, Danzmann RG: Comparative Genome Analysis of the Primary Sex-Determining Locus in Salmonid Fishes. Genome research 2003, I3(2):272-280.

17. Ng SH, Artieri CG, Bosdet IE, Chiu R, Danzmann RG, Davidson WS, Ferguson MM, Fjell CD, Høyheim B, Jones SJ, de Jong PJ, Koop BF, Krzywinski MI, Lubieniecki K, Marra MA, Mitchell LA, Mathewson C, Osoegawa K, Parisotto SE, Phillips RB, Rise ML, von Schalburg KR, Schein JE, Shin H, Siddiqui A, Thorsen J, Wye N, Yang G, Zhu B: A physical map of the genome of Atlantic salmon, Salmo salar. Genomics 2005, 86:396-404.

18. Hayes B, Lærdahl JK, Lien S, Moen T, Berg P, Hindar K, Davidson WS, Koop BF, Adzhubei A, Høyheim B: An extensive resource of single nucleotide polymorphism markers associated with Atlantic salmon (Salmo salar) expressed sequences. Aquaculture 2007, 265:82-90.

19. Hayes BJ, Nilsen K, Berg PR, Grindflek E, Lien S: SNP detection exploiting multiple sources of redundancy in large EST col- 
lections improves validation rates. Bioinformatics 2007 , 23:1692-1693.

20. Slate J, Pemberson JM: Admixture and patterns of linkage disequilibrium in a free-living vertebrate population. Journal of Evolutionary Biology 2007, 20: I415-1427.

21. Abecasis GR, Noguchi E, Heinzmann A, Traherne JA, Bhattacharyya S, Leaves NI, Anderson GG, Zhang Y, Lench NJ, Carey A, Cardon LR, Moffatt MF, Cookson WOC: Extent and Distribution of Linkage Disequilibrium in Three Genomic Regions. American journal of human genetics 200I, 68(I): 19I-197.

22. Reich DE, Cargill M, Bolk S, Ireland J, Sabeti PC, Richter DJ, Lavery T, Kouyoumjian R, Farhadian SF, Ward R, Lander ES: Linkage disequilibrium in the human genome. Nature 200I, 4I I (6834): 199-204.

23. Farnir F, Coppieters W, Arranz J], Berzi P, Cambisano N, Grisart B, Karim L, Marcq F, Moreau L, Mni M, Nezer C, Simon P, Vanmanshoven P, Wagenaar D, Georges M: Extensive Genome-wide Linkage Disequilibrium in Cattle. Genome Research 2000 10:220-227.

24. Nsengimana J, Baret P, Haley CS, Visscher PM: Linkage Disequilibrium in the Domesticated Pig. Genetics 2004, I 66:1395- I 404

25. Lindblad-Toh K, Wade CM, Mikkelsen TS, Karlsson EK, Jaffe DB, Kamal M, Clamp M, Chang JL, Kulbokas EJ, Zody MC, Mauceli E, Xie $X$, Breen M, Wayne RK, Ostrander EA, Ponting CP, Galibert F, Smith DR, dejong PJ, Kirkness E, Alvarez P, Biagi T, Brockman W, Butler J, Chin CW, Cook A, Cuff J, Daly MJ, Decaprio D, Gnerre S, Grabherr M, Kellis M, Kleber M, Bardeleben C, Goodstadt L, Heger A, Hitte C, Kim L, Koepfli KP, Parker HG, Pollinger JP, Searle SMJ, Sutter NB, Thomas R: Genome sequence, comparative analysis and haplotype structure of the domestic dog. Nature 2005, 438(7069):745-746

26. Backström N, Qvarnström A, Gustafsson L, Ellegren $\mathrm{H}$ : Levels of linkage disequilibrium in a wild bird population. Biology Letters 2006, 2:435-438.

27. Hartley SE, Horne MT: Chromosome polymorphism and constitutive heterochromatin in the Atlantic salmon, Salmo salar. Chromosoma 1984, 89:377-380.

28. Davisson MT, Wright JE, Atherton LM: Cytogenetic analysis of pseudolinkage of LDH loci in the teleost genus Salvelinus. Genetics 1973, 73:645-658.

29. Moen T, Sonesson AK, Hayes B, Lien S, Munck H, Meuwissen THE: Mapping of a quantitative trait locus for resistance against infectious salmon anaemia in Atlantic salmon (Salmo Salar): comparing survival analysis with analysis on affected/resistant data. BMC Genetics 2007, 8:53.

30. Thorgaard GH, Allendorf FW, Knudsen KL: Gene-centromere mapping in rainbow trout: High interference over long map distances. Genetics 1983, 103:77I-783.

31. Streisinger GF, Singer F, Walker C, Knauber D, Dower N: Segregation analyses and gene-centromere distances in zebrafish. Genetics 1986, I | 2:3 ||-319.

32. Allendorf FW, Seeb JE, Knudsen KL, Thorgaard GH: Gene-centromere mapping of $\mathbf{2 5}$ loci in rainbow trout. J Hered 1986, 77:307-312.

33. Lindner KR, Seeb JE, Habicht C, Knudsen KL, Kretschmer E: Genecentromere mapping of 312 loci in pink salmon by half-tetrad analysis. Genome 2000, 43:538-549.

34. Gilbey J, Verspoor E, Mo TA, Sterud E, Olstad K, Hytterød S, Jones $C$, Noble L: Identification of genetic markers associated with Gyrodactylus salaris resistance in Atlantic salmon Salmo salar. Dis Aquat Organ 2006, 71:119-129.

35. Sham PC, Zhao JH, Curtis D: The effect of marker characteristics on the power to detect linkage disequilibrium due to single or multiple ancestral mutations. Annals of Human Genetics 2000, 64(2): $161-169$

36. Varilo T, Paunio T, Parker A, Perola M, Meyer J, Terwilliger JD, Peltonen $L$ : The interval of linkage disequilibrium (LD) detected with microsatellite and SNP markers in chromosomes of Finnish populations with different histories. Human Molecular Genetics 2003, I2(1):51-59.

37. Nordborg M, Tavaré S: Linkage disequilibrium: what history has to tell us. Trends Genet 2002, 18:83-89.

38. Teare M, Dunning A, Durocher F, Rennart G, Easton D: Sampling distribution of summary linkage disequilibrium measures. Annals of Human Genetics 2002, 66(3):223-233.
39. Gjedrem T, Gjøen HM, Gjerde B: Genetic origin of Norwegian farmed Atlantic salmon. Aquaculture 199I, 98:4I-50.

40. Kruglyak L: Prospects for whole-genome linkage disequilibrium mapping of common disease genes. Nature genetics 1999 , 22(2): 139-144

4I. Ardlie KG, Kruglyak L, Seielstad M: Patterns of linkage disequilibrium in the human genome. Nature Reviews Genetics 2002 3:299-309.

42. CIGENE [http://www.cigene.no]

43. Ewing B, Hillier L, Wendl CW, Green P: Base-calling of automated sequencer traces using Phred. I. Accuracy assessment. Genome Research 1998, 8:175-185.

44. Marth G T, Korf I, Yandell MD, Yeh RT, Gu Z, Zakeri H, Stitzial NO, Hillier L, Kwok PY, Gish WR: A general approach to singlenucleotide polymorphism discovery. Nature Genetics 1999, 23:452-456.

45. Rise ML, von Schalburg KR, Brown GD, Mawer MA, Devlin RH, Kuipers N, Busby M, Beetz-Sargent M, Alberto R, Gibbs AR, Hunt P, Shukin R, Zeznik JA, Nelson C, Jones SRM, Smailus DE, Jones SJM, Schein JE, Marra MA, Butterfield YSN, Stott JM, Ng SHS, Davidson WS, Koop BF: Development and Application of a Salmonid EST Database and cDNA Microarray: Data Mining and Interspecific Hybridization Characteristics. Genome Research 2004 I 4:478-490.

46. Salmon Genome Project [http://www.salmongenome.no]

47. Sequenom [http://www.sequenom.com]

48. Tang K, Fu DJ, Julien D, Braun A, Cantor CR, Köster H: Chip-based genotyping by mass spectrometry. Proc Natl Acad Sci USA 1999 , 96:10016-10020

49. Freedman D, White SJ, Potter S, Eichler EE, Den Dunnen JT, Brookes AJ: Complex SNP-related sequence variation in segmental genome duplications. Nat Genet 2004, 36:86I-866.

50. CGRASP [http://www.uvic.ca/cbr/grasp]

51. Stam P, Van Ooijen JW: JoinMap (tm) version 2.0: Software for the calculation of genetic linkage maps. CPRO-DLO, Wageningen, The Netherlands; 1995.

52. Abecasis GR, Cookson WO: GOLD--graphical overview of linkage disequilibrium. Bioinformatics 2000, 16:182-183.

53. Cramer H: Mathematical Models of Statistics. Princeton, New Jersey, USA, Princeton University Press; 1946.

54. Hill WG: Estimation of effective population size from data on linkage disequilibrium. Genetical Research 1981, 38:209-216..

55. Sved JA: Linkage disequilibrium and homozygosity of chromosome segments in finite populations. Theoretical Population Biology |97|, 2:|24-|4|.

Publish with BioMed Central and every scientist can read your work free of charge

"BioMed Central will be the most significant development for disseminating the results of biomedical research in our lifetime. "

Sir Paul Nurse, Cancer Research UK

Your research papers will be:

- available free of charge to the entire biomedical community

- peer reviewed and published immediately upon acceptance

- cited in PubMed and archived on PubMed Central

- yours - you keep the copyright

BioMedcentral 